\title{
Resin Flow, Symptom Development, and Lignin Biosynthesis of Two Pine Species in Response to Wounding and Inoculation with Fusarium circinatum
}

\author{
Ki Woo Kim*, In Jung Lee ${ }^{2}$, Chang Soo Kim³ ${ }^{3}$ In-Yong Eom ${ }^{4}$, Joon-Weon Choi ${ }^{4}$, Don Koo Lee ${ }^{4}$ and \\ Eun Woo Park ${ }^{5 *}$ \\ ${ }^{1}$ National Instrumentation Center for Environmental Management, Seoul National University, Seoul 151-921, Korea \\ ${ }^{2}$ Research Institute for Agriculture and Life Sciences, Seoul National University, Seoul 151-921, Korea \\ ${ }^{3}$ Department of Forest Genetic Resources, Korea Forest Research Institute, Suwon 441-350, Korea \\ ${ }^{4}$ Department of Forest Sciences, Seoul National University, Seoul 151-921, Korea \\ ${ }^{5}$ Department of Agricultural Biotechnology, Seoul National University, Seoul 151-921, Korea \\ (Received on July 14, 2010; Accepted on November 10, 2010)
}

Resin flow, symptom development, and lignin biosynthesis in response to wounding and fungal inoculation were investigated in Pinus rigida and Pinus densiflora. The two-year-old seedling stems were subjected to three types of treatments: (i) wounding without inoculation, (ii) wound-inoculation with a conidial suspension of Fusarium circinatum, and (iii) pre-wounding woundinoculation with the fungus 20 days after the initial wounding. Resin flow from wounding sites was more evident in $P$. rigida than $P$. densiflora in all treatments. The wound-inoculation with the fungus induced almost two-fold higher levels of resin flow than the other treatments in both species. The pre-wounding woundinoculation appeared to result in a decrease in pitch canker development in the two pine species. Some reductions in disease severity were observed in the prewounding wound-inoculated $P$. rigida, showing a mean disease severity of less than $85 \%$, compared with approximately $100 \%$ in the wound-inoculated stems. Disease severity was approximately $50 \%$ in the woundinoculated $P$. densiflora, whereas $10 \%$ in the pre-wounding wound-inoculated stems. Higher amounts of lignin were found from bark (ca. 40\%) than from xylem (ca. $30 \%$ ). The wound-inoculated bark and the pre-wounding wound-inoculated bark exhibited higher amounts of lignin among the other treatments. These results suggest that the wound-inoculation apparently prompt the increase in resin flow and lignin biosynthesis from the two pine species, and the prior wounding may be involved in decreased disease severity against the further invasion of $F$. circinatum.

\footnotetext{
*Corresponding authors.

KW Kim

Phone) +82-2-880-4957, FAX) +82-2-888-4847

E-mail)kiwoo@snu.ac.kr

EW Park

Phone) +82-2-880-4672, FAX) +82-2-873-2317

E-mail)ewpark@snu.ac.kr
}

Keywords : Fusarium circinatum, inoculation, lignin, pitch canker, resin, wound

Coniferous trees (conifers) have colonized the earth's landmass to a greater extent than any other groups of plants (Phillips and Croteau, 1999). Effective defense strategies are part of the key to their successful colonization of diverse habitats from alpine and arctic timberlines to subtropical swamps (Franceschi et al., 2005). Conifers are all characterized by secondary growth, which gives a complicating factor of more complex growth than annual angiosperms commonly used in most host-pathogen studies (Hammerschmidt, 2006). Exposed to mechanical injury and attacks by numerous pathogens and insects, conifers deploy various defense mechanisms as other plants (Luchi et al., 2005). Constitutive production of either structural defense products such as cuticle and periderm or biochemical defense products such as phenolics and terpenoids provides a generalized defense against a broad range of organisms (Franceschi et al., 2005). Synthesized in specialized secretory structures called resin cells and resin ducts, resin is known to act primarily as a wound cleanser and sealant, and also shown to have antimicrobial activity (Luchi et al., 2005). Oleoresin (a mixture of monoterpenes, resin acids, and neutral compounds) is the most obvious and widespread resin of coniferous trees, providing a chemical and physical barrier against biotic intrusions (Lombardero et al., 2000, 2006). Terpenoids in pine resin are considered to readily interact with cell membranes, which can lead to uncontrolled cell leakage, consequently resulting in cell death of insects (Mumm and Hilker, 2006).

Pitch canker is one of the most destructive fungal diseases of pines worldwide (Viljoem et al., 1994; Wingfield et al., 2002). The causal agent is known as a necrotrophic fungus Fusarium circinatum Nirenberg and O'Donnell (teleomorph Gibberella circinata Nirenberg and O'Donnell) 
(Correll et al., 1991; Nirenberg and O'Donnell, 1998). The initial symptoms of the disease include wilting of needles and resin flow from the infected areas (Kim et al., 2008). Different levels of resistance to the disease were reported among pine species: pitch pine (Pinus rigida P. Mill.) as a susceptible species and Japanese red pine ( $P$. densiflora Sieb. \& Zucc.) as a resistant one (Lee et al., 2000). It is widely accepted that the fungal pathogen enters host tissues through wounds caused by insect feeding, silvicultural practices, or weather-related damages (Gordon, 2006). A genomics approach (Morse et al., 2004) showed that pine genes regulated by $F$. circinatum were distinct from those incited by biotrophic pathogens. Recently, an electron microscopic study (Kim et al., 2009) showed the wound periderm and hyphal ultrastructure in $P$. rigida and $P$. densiflora stems inoculated with $F$. circinatum. However, little information is available on the differences in resin flow, symptom development, and lignin content between susceptible and resistant pine species in response to wounding and inoculation with $F$. circinatum. In this study, responses of $P$. rigida and $P$. densiflora to wounding and pathogen inoculation were investigated, and different levels of disease severity by prior wounding were examined in the pine- $F$. circinatum interaction.

\section{Materials and Methods}

Plant materials. Two pine species, $P$. rigida and $P$. densiflora, were prepared as reported previously (Thoungchaleun et al., 2008). The two-year-old seedlings were transferred from a nursery to a growth chamber maintained at $25^{\circ} \mathrm{C}$. Stems of $10 \mathrm{~mm}$ in diameter were used for inoculation with F. circinatum (Kim et al., 2009).

Inoculum preparation. The $F$. circinatum culture was prepared according to the method previously reported (Thoungchaleun et al., 2008). Briefly, the isolate was grown on potato dextrose agar (PDA) plates at $25^{\circ} \mathrm{C}$ with a $12 \mathrm{~h}$ photoperiod a day for one week. The PDA plates were flooded with sterilized distilled water and scraped to make a conidial suspension, which was adjusted to a concentration of $1.0 \times 10^{7}$ microconidia/ml using a hemacytometer.

Wounding/inoculation treatments. In order to obtain the net wounding effects on host responses to pathogen infection, each pine species was treated in three different ways as follows: (i) Wound only; three wounds (each ca. $5 \times 5 \mathrm{~mm}^{2}$ with $1 \mathrm{~mm}$ depth of underlying tissues) were made using a razor blade on each seedling along the stem. Without inoculation, the wounded seedlings were kept to the growth chamber maintained at $25^{\circ} \mathrm{C}$ under the relative humidity of $60 \%$ for 90 days. (ii) Wound-inoculation; stems were wounded as described above, and then a drop $(100 \mu \mathrm{l})$ of the conidial suspension was placed on each wounding site. Immediately after inoculation, the seedlings were also maintained in the growth chamber as mentioned above. (iii) Pre-wounding wound-inoculation; stems were wounded as described above. After 20 days of the initial wounding, three additional wounds (ca. $3 \mathrm{~cm}$ apart from the initial wounds) were made on the stems, and then a drop of the conidial suspension was placed on the newly wounding sites. The wound-inoculated seedlings were kept in the growth chamber. Untreated seedlings of both species were also included as a control.

Resin flow and disease severity. Resin flow and disease severity on the pine species were measured using modified methods from relevant studies (Barrows-Broaddus and Dwinell, 1983; Kim et al., 2008). The levels of resin flow from the wounding sites (each ca. $5 \times 5 \mathrm{~mm}^{2}$ ) were visually assessed as the percent of resin-covered areas in wounding sites from 5 to 45 days after inoculation. Disease severity was rated from 45 to 95 days after inoculation according to a scale where $0=$ no disease symptoms, $25 \%=$ weak blight symptoms on stems and barely visible needle browning, $50 \%=$ clear stem blight and needle browning symptoms, $75 \%=$ extensive stem blight and needle browning, and $100 \%=$ extreme stem blight and needle browning. One seedling was allocated for each wounding/inoculation treatment. The experiment was arranged as a completely randomized design with three replications, and it was repeated twice. Data from two experiments were pooled to compare mean areas covered with resin and mean disease severity among the treatments by Fisher's protected least significant difference (FLSD) test using a statistical software (SAS; SAS Institute, Cary, NC, USA).

Determination of lignin content. Stems of both pine species under the three wounding/inoculation treatments were collected 45 days after each treatment and air dried at room temperature $\left(20\right.$ to $\left.24^{\circ} \mathrm{C}\right)$ for one week for lignin quantification. Unwounded stems of both species were also included as a control. The air dried specimens were divided into bark and xylem, and finely ground with a laboratory ball miller (Pulverisette 6; Fritsch, Idar-Oberstein, Germany). Lignin content in the specimens was determined as reported previously (Sluiter et al., 2008). Briefly, the ground materials were subjected to the first acid hydrolysis step using $72 \%$ $(\mathrm{w} / \mathrm{w})$ aqueous sulfuric acid at $30^{\circ} \mathrm{C}$ with occasional stirring for $1 \mathrm{~h}$. The sulfuric acid hydrolysates were immediately diluted with distilled water to $4 \%$ and autoclaved at $121^{\circ} \mathrm{C}$ for $40 \mathrm{~min}$ as the second acid hydrolysis step. After the total hydrolysates were cooled down to room temperature, the hydrolysis residue (acid insoluble lignin) was 
filtered and weighed after dryness at $105^{\circ} \mathrm{C}$. The quantity of lignin was expressed as the percent of the dry weight of total hydrolysates. This experiment was conducted with two replications and repeated twice. Mean lignin content was compared among the treatments as described above.

\section{Results}

Resin flow from wounding sites. Wounding sites turned brown after wounding on P. rigida stems (Fig. 1A-1C). Copious resin flowed from wound margins, covered the
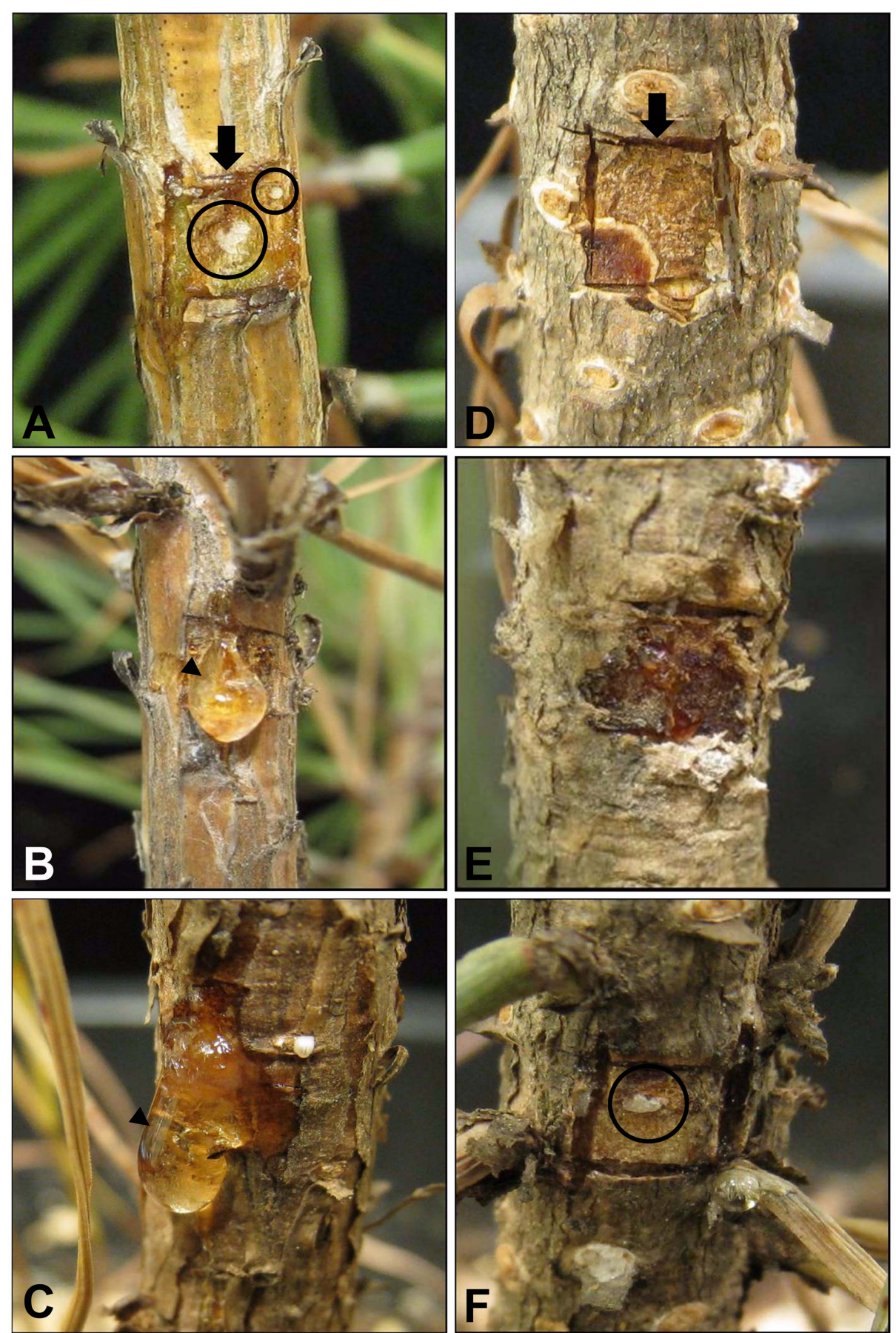

Fig. 1. Wounded stems of P. rigida (A-C) and P. densiflora (D-F). (A and D) Wounded stems after 20 days. Most wounding sites (arrows) turned brown. Resin solidified as white remains (circles). (B and E) Wound-inoculated stems 20 days after inoculation. Copious resin flow (an arrowhead) was more evident in $P$. rigida than $P$. densiflora. (C and F) Pre-wounding wound-inoculated stems 20 days after inoculation. An arrowhead and a circle indicate resin flow and white remains on the stems, respectively. 

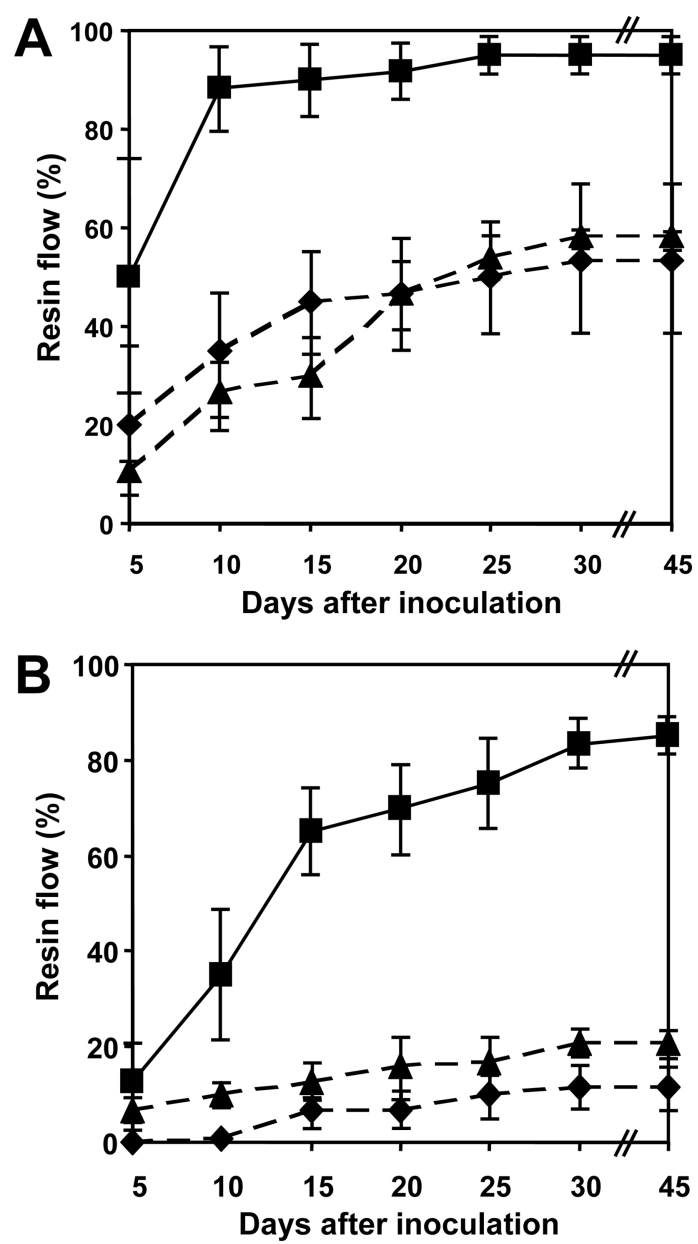

Fig. 2. Temporal progress of resin flow from initially wounding sites of $P$. rigida (A) and $P$. densiflora (B). Resin flow from the wound-inoculated stems was significantly higher than the other treatments throughout the experiment period in both species $(P=0.01) .(\$=$ Wounded stems; $\boldsymbol{\square}=$ Wound-inoculated stems; $\boldsymbol{\Delta}=$ Pre-wounding wound-inoculated stems. Only initially wounding sites were monitored.)

wounding sites, and solidified as white remains. The resin usually appeared to be transparent or whitish to yellow. Similar results were observed from $P$. densiflora stems (Fig. 1D-1F).

Different levels of resin flow were found in P. rigida stems among the wounding/inoculation treatments (Fig. 2A). The wound-inoculated stems produced resin flow approximately twice as much as stems of the other treatments 10 days after inoculation. The other treatments showed less than $60 \%$ of resin-covered areas 45 days after inoculation, significantly lower than the wound-inoculated stems $(P=$ $0.01)$. There were no significant differences in resin flow between the stems with wounding and pre-wounding wound inoculation throughout the experiment $(P=0.01)$.

It was apparent that $P$. densiflora stems produced less

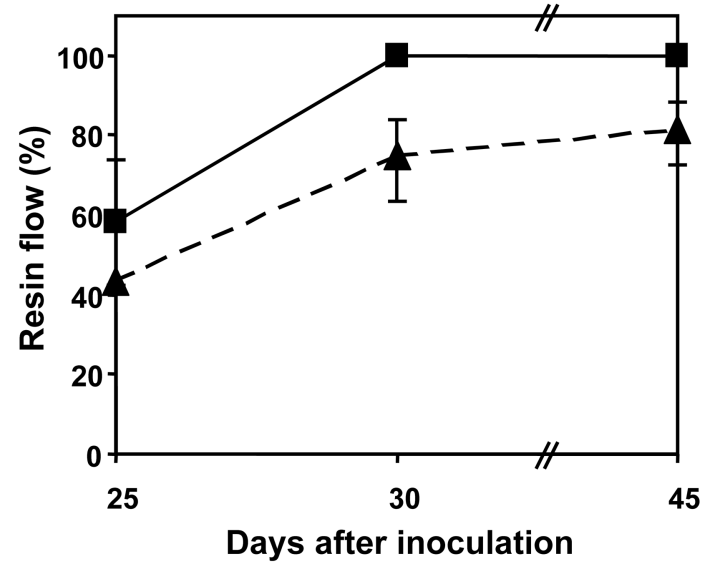

Fig. 3. Temporal progress of resin flow from newly wounding sites of the pre-wounding wound-inoculated stems of $P$. rigida $(\boldsymbol{\square})$ and $P$. densiflora $(\boldsymbol{\Delta})$. Lower amount of resin flow was observed from the stems of $P$. densiflora than those of $P$. rigida throughout the experiment $(P=0.01)$.

amount of resin than $P$. rigida stems from initially wounding sites in all treatments throughout the experiment (Fig. 2B). Wound-inoculated stems exhibited a great increase in resin flow, reaching to over $80 \%$ of resin-covered areas 45 days after inoculation. Significant differences were found in resin flow between the wound-inoculated stems and the other treatments $(P=0.01)$. Neither marked increase nor differences in resin flow were observed in the other treatments throughout the experiment, which showed mostly below $20 \%$ of resin-covered areas $(P=0.01)$.

The pre-wounding wound-inoculation also showed resin flow from newly wounding sites, which was similar to those of other wounding/inoculation treatments (Fig. 3). The $P$. rigida stems showed approximately $60 \%$ resin flow 25 days after inoculation, resulting in 100\% 30 days after inoculation. Significantly less amount of resin flow was observed from the stems of $P$. densiflora than those of $P$. rigida throughout the experiment, ranging from around $40 \%$ to $80 \%$ of resin-covered areas $(P=0.01)$.

Disease severity. There were different levels of disease severity in the two pine species among the wounding/ inoculation treatments. Wounded stems without inoculation appeared healthy (Fig. 4A and 4D). However, wound-inoculated stems were greatly damaged, exhibiting extensive stem blight and needle blight (Fig. 4B and 4E). Stem blight and needle browning were also observed in the pre-wounding wound-inoculated stems (Fig. 4C and 4F).

Mean disease severity was approximately $100 \%$ in the wound-inoculated $P$. rigida stems 45 days after inoculation (Fig. 5A). Reductions in disease severity were found in the pre-wounding wound-inoculated stems, showing a mean disease severity of less than $80 \%$. There was a gradual 

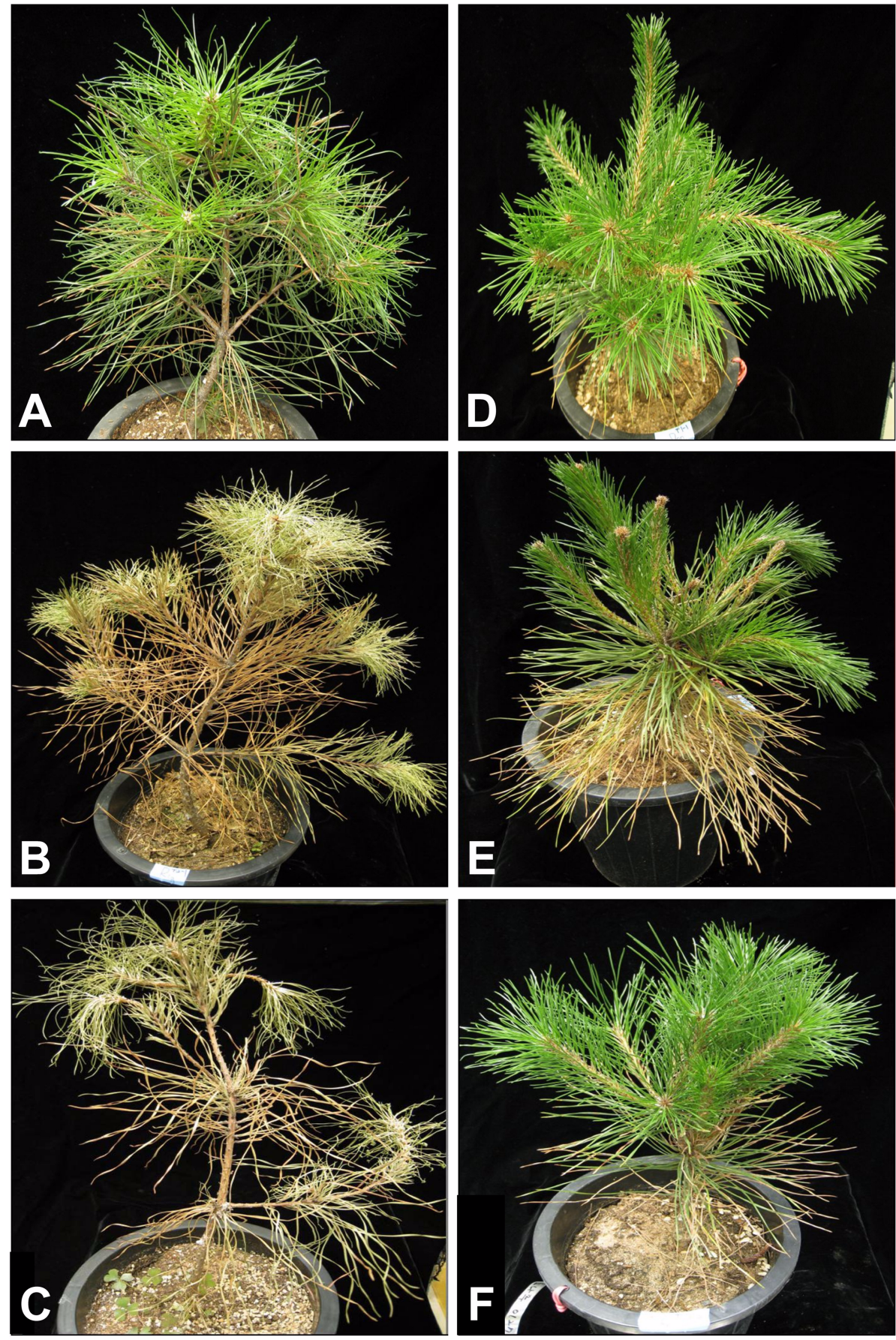

Fig. 4. Comparison of pitch canker development between $P$. rigida (A-C) and $P$. densiflora (D-F). (A and D) Wounded stems after 45 days. They appeared healthy in both species. (B and E) Wound-inoculated stems 45 days after inoculation. Extensive blight of stem and needle was noted in P. rigida (B). The blight was less severe in P. densiflora (E) than P. rigida. (C and F) Pre-wounding wound-inoculated stems 25 days after wound-inoculation. They also showed blight of stem and needle in both species.

increase in disease severity of the pre-wounding woundinoculated stems, reaching to $100 \%$ disease severity 75 days after inoculation. The stems with wounding alone exhibited minor needle browning.

Relatively low levels of disease severity were found in stems of $P$. densiflora in all treatments as compared with $P$. rigida (Fig. 5B). Disease severity was approximately $50 \%$ in the wound-inoculated stems 45 days after inoculation. With higher variations of disease severity than that of $P$. rigida, the wound-inoculated stems of $P$. densiflora showed 

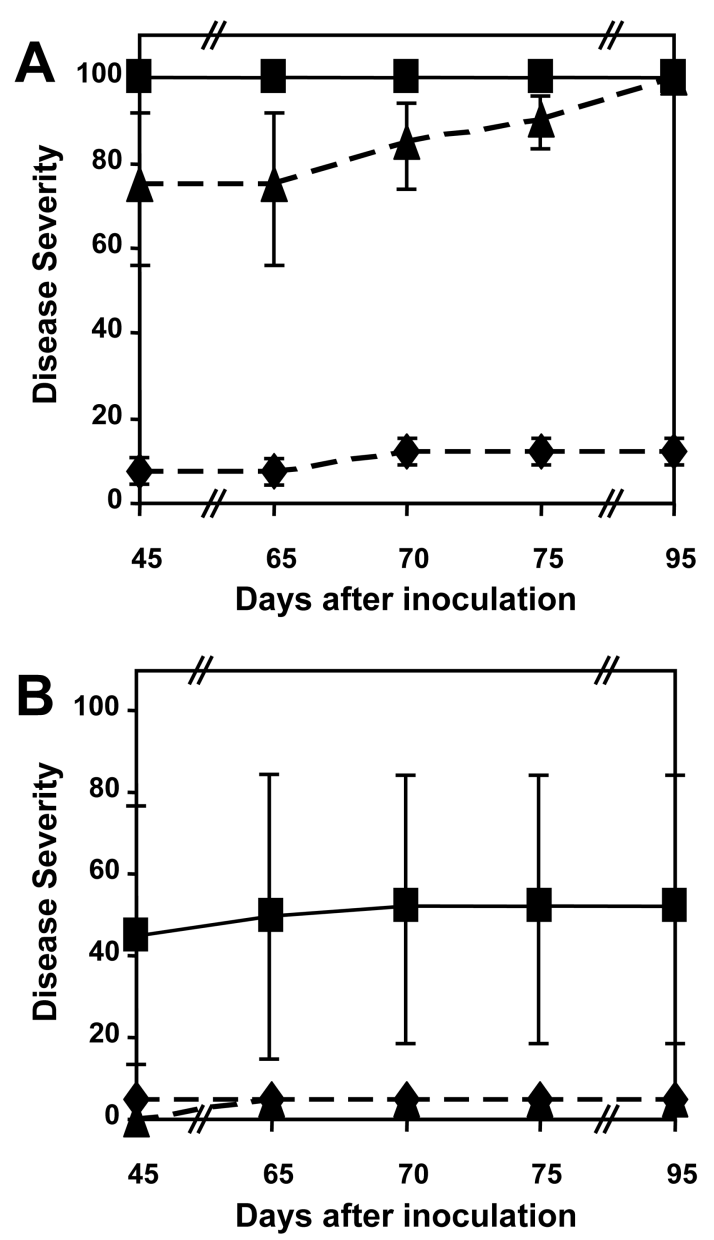

Fig. 5. Temporal progress of pitch canker development in $P$. rigida (A) and $P$. densiflora (B). The stems with wounding alone showed minor needle browning. Reductions in disease severity were found in the pre-wounding wound-inoculated stems in both species. ( $\boldsymbol{\nabla}=$ Wounded stems; $\boldsymbol{\nabla}=$ Wound-inoculated stems; $\boldsymbol{\Delta}=$ Pre-wounding wound-inoculated stems)

slight increase in disease severity (ca. 53\%) throughout the experiment. Approximately $5 \%$ disease severity was recorded from the pre-wounding wound-inoculated stems.

Lignin content from bark and xylem. It was obvious in the two pine species that higher amounts of lignin were found from bark (ca. 40\%) than from xylem (ca. 30\%) (Fig. 6). The wound-inoculated bark of $P$. rigida exhibited the highest amounts of lignin as compared with the other treatments $(P=0.01)$. In $P$. densiflora bark, lignin contents of either the wound-inoculated stems or the pre-wounding wound-inoculated stems were significantly higher than those of the wounded stems and the control $(P=0.01)$. No significant differences in lignin content from xylem were observed between the pine species regardless of the wounding/inoculation treatments $(P=0.01)$.

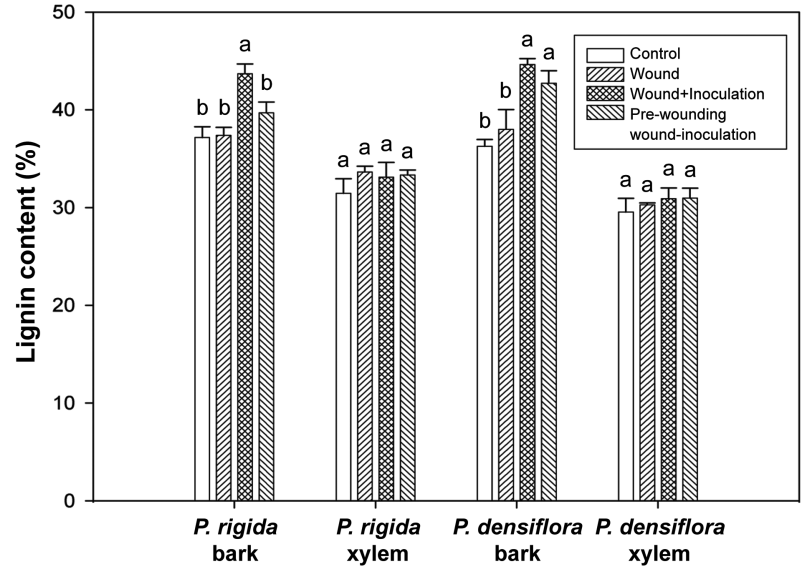

Fig. 6. Lignin content from bark and xylem of $P$. rigida and $P$. densiflora. Bars in the columns indicate the standard deviations. Higher amounts of lignin were found from bark than from xylem in the two species. The wound-inoculated bark and the prewounding wound-inoculated stems exhibited the highest amounts of lignin as compared with the other treatments. The same letters denote no significant difference at $P=0.01$ according to FLSD test.

\section{Discussion}

This study demonstrated the resin flow, symptom development, and lignin biosynthesis of $P$. rigida and $P$. densiflora seedling stems in response to wounding and inoculation with $F$. circinatum. Different levels of resin flow and disease severity were evident in the two pine species. The high resin content of $P$. rigida, a commonly known species characteristic underlying the common name pitch pine (Esteban et al., 2005), is considered to be responsible for more copious resin flow from stem wounds than $P$. densiflora. Constitutive resin stores, not from traumatic resin duct systems, of $P$. rigida examined in this study are assumed to be involved in the response of pines to mechanical wounding, although $P$. rigida is susceptible to pitch canker. Interspecific hybrids of $P$. rigida also showed higher amounts of resin flow from stems than $P$. densiflora when inoculated with the fungus (Kim et al., 2008). These results imply that constitutive resin stores are not essential for conferring resistance to pine species against $F$. circinatum. An intriguing finding in this study was the almost twofold higher levels of resin-covered areas from the woundinoculated stems than other treatments regardless of pine species. A similar instance was noted in loblolly pine $(P$. taeda L.) where wounding with inoculation resulted in a greater resin flow than wounding alone (Knebel et al., 2008). In Austrian pine (P. nigra Arn.), fluctuations of resin flow from wounding sites were attributed to different morphogenetic features of the traumatic resin duct system according to different wounding/inoculation treatments 
(Luchi et al., 2005).

Concomitantly, the results of lignin content were consistent with those of resin flow from the two pine species, showing a higher increase in lignin content from the woundinoculated bark and the pre-wounding wound-inoculated bark than any other treatments. The increase in lignin content from the bark of $P$. densiflora may be associated with the severity reduction in the pre-wounding woundinoculated stems. Given the insignificant changes of lignin content in xylem after the wounding/inoculation treatments, the biosynthesis of lignin through the wounding/inoculation appears to be mostly localized to bark, reinforcing lignocellulosic architecture around wounding sites. The identification of triggering factors governing the increases in resin flow and lignin content, e.g., live pathogens, (nonpathogenic) microbes, etc., needs to be addressed in further studies. To our knowledge, this is the first report on the quantitative analysis of lignin content in the bark and xylem of $P$. rigida and $P$. densiflora seedling stems according to wounding/inoculation treatments.

Other differences between the two pine species were found in pitch canker development after the wounding and inoculation. The disease reduction by prior wounding appeared to be more operative in $P$. densiflora than $P$. rigida, showing a distinct decrease in disease severity. Meanwhile, the disease reduction in $P$. rigida under the pre-wounding wound-inoculation would be characterized by delayed disease development, prolonging incubation period of the inoculated hosts. The results from this study suggest that prior wounding may be partially contributing to decreased pitch canker development. For trees subjected to branch removal prior to inoculation, the role of wounding was shown to have no significant effects on disease reduction in native stands of P. muricata (Schmale and Gordon, 2003). The controversy about wounding effects on disease reduction may reflect differential effects of the degrees or intensity of wounding on host resistance, coupled with host lines and pathogen virulence.

In conifers, the decreased disease development by prior inoculation was first reported from Monterey pine (Pinus radiata D. Don), showing enhanced resistance of trees inoculated with a pathogenic strain of $F$. circinatum to subsequent challenge inoculations (Bonello et al., 2001). While previous studies (Bonello et al., 2001, 2006; Schmale and Gordon, 2003; Woo et al., 2005) focused specifically on the roles of pathogens in decreased disease severity of trees, the wounding/inoculation treatments in this study could allow the assessment of wounding effects on decreased disease severity in pines. It is hypothesized that the pine seedlings become less vulnerable to subsequent inoculations after an initial wounding than unwounded seedlings. Both salicylic and jasmonic acids have been suggested as inducers of decreased disease development in conifers, as verified when applied exogenously in high concentrations (Davis et al., 2002; Hudgins et al., 2004).

In summary, the wound-inoculation apparently prompted the increase in resin flow and lignin biosynthesis from stems of $P$. rigida and $P$. densiflora, and the prior wounding may have been involved in decreased disease severity against the further invasion of $F$. circinatum. Elucidation of mechanisms underlying wound responses in conifers will yield a venue for a comprehensive understanding of tree disease management for sustainable forest ecosystem.

\section{Acknowledgments}

This study was carried out with the support of 'Forest Science \& Technology Projects (Project No. S210608L 0101704C)' provided by Korea Forest Service.

\section{References}

Barrows-Broaddus, J. and Dwinell, L. D. 1983. Histopathology of Fusarium moniliforme var. subglutinans in four species of southern pines. Phytopathology 73:882-889.

Bonello, P., Gordon, T. R. and Storer, A. J. 2001. Systemic induced resistance in Monterey pine. For. Path. 31:99-106.

Correll, J. C., Gordon, T. R., McCain, A. H., Fox, J. W., Koehler, C. S., Wood, D. L. and Schultz, M. E. 1991. Pitch canker disease in California: pathogenicity, distribution, and canker development on Monterey pine (Pinus radiata). Plant Dis. 75:676-682.

Davis, J. M., Wu, H., Cooke, J. E. K., Reed, J. M., Luce, K. S. and Michler, C. H. 2002. Pathogen challenge, salicylic acid, and jasmonic acid regulate expression of chitinase gene homologs in pine. Mol. Plant Microbe Interact. 15:380-387.

Esteban, L. G., Gasson, P., Climent, J. M., De Palacios, P. and Guindeo, A. 2005. The wood of Pinus canariensis and its resinous heartwood. IAWA J. 26:69-77.

Gordon, T. R. 2006. Pitch canker disease of pines. Phytopathology 96:657-659.

Franceschi, V. R., Krokene, P., Christiansen, E. and Krekling, T. 2005. Anatomical and chemical defenses of conifer bark against bark beetles and other pests. New Phytol. 167:353-376.

Hammerschmidt, R. 2006. Host-pathogen interaction in conifers: Complicated systems yield interesting possibilities for research. Physiol. Mol. Plant Pathol. 68:93-94.

Hudgins, J. W., Christiansen, E. and Franceschi, V. R. 2004. Induction of anatomically based defense responses in stems of diverse conifers by methyl jasmonate: a phylogenetic perspective. Tree Physiol. 24:251-264.

Kim, K. W., Lee, I. J., Thoungchaleun, V., Kim, C. S., Lee, D. K. and Park, E. W. 2009. Visualization of wound periderm and hyphal profiles in pine stems inoculated with the pitch canker fungus Fusarium circinatum. Microsc. Res. Techniq. 72:965973. 
Kim, Y.-S., Woo, K.-S., Koo, Y.-B. and Yeo, J.-K. 2008b. Variation in susceptibility of six pine species and hybrids to pitch canker caused by Fusarium circinatum. For. Path. 38:419428.

Knebel, L., Robinson, D. J., Wentworth, T. R. and Klepzig, K. D. 2008. Resin flow responses to fertilization, wounding and fungal inoculation in loblolly pine (Pinus taeda) in North Carolina. Tree Physiol. 28:847-853.

Lee, J. K., Lee, S.-H., Yang, S.-I. and Lee, Y.-W. 2000. First report of pitch canker disease on Pinus rigida in Korea. Plant Pathol. J. 16:52-54.

Lombardero, M. J., Ayres, M. P. and Ayres, B. D. 2006. Effects of fire and mechanical wounding on Pinus resinosa resin defenses, beetle attacks, and pathogens. For. Ecol. Manage. 225:349-358.

Lombardero, M. J., Ayres, M. P., Lorio, P. L. Jr. and Ruel, J. J. 2000. Environmental effects on constitutive and inducible resin defences of Pinus taeda. Ecol. Lett. 3:329-339.

Luchi, N., Ma, R., Capretti, P. and Bonello, P. 2005. Systemic induction of traumatic resin ducts and resin flow in Austrian pine by wounding and inoculation with Sphaeropsis sapinea and Diplodia scrobiculata. Planta 221:75-84.

Morse, A. M., Nelson, C. D., Covert, S. F., Holliday, A. G., Smith, K. E. and Davis, J. M. 2004. Pine genes regulated by the necrotrophic pathogen Fusarium circinatum. Theor. Appl. Genet. 109:922-932.

Mumm, R. and Hilker, M. 2006. Direct and indirect chemical defence of pine against folivorous insects. Tren. Plant Sci. 11:351-358.
Nirenberg, H. I. and O’Donnell, K. 1998. New Fusarium species and combinations within the Gibberella fujikuroi species complex. Mycologia 90:434-458.

Phillips, M. A. and Croteau, R. B. 1999. Resin-based defenses in conifers. Tren. Plant Sci. 4:184-190.

Schmale, D. G. and Gordon, T. R. 2003. Variation in susceptibility to pitch canker disease, caused by Fusarium circinatum, in native stands of Pinus muricata. Plant Pathol. 52:720-725.

Sluiter, A., Hames, B., Ruiz, R., Scarlata, C., Sluiter, J., Templeton, D. and Crocker, D. 2008. Determination of structural carbohydrates and lignin in biomass. In: Laboratory Analytical Procedure (LAP). pp. 1-16. National Renewable Energy Laboratory, Golden, USA.

Thoungchaleun, V., Kim, K. W., Lee, D. K., Kim, C. S. and Park, E. W. 2008. Pre-infection behavior of the pitch canker fungus Fusarium circinatum on pine stems. Plant Pathol. J. 24:112117.

Viljoem, A., Wingfield, M. J. and Marasas, W. F. O. 1994. First report of Fusarium subglutinans f. sp. pini on pine seedlings in South Africa. Plant Dis. 78:309-312.

Wingfield, M. J., Jacobs, A., Coutinho, T. A., Ahumada, R. and Wingfield, B. D. 2002. First report of the pitch canker fungus, Fusarium circinatum, on pines in Chile. Plant Pathol. 51:397.

Woo, K. S., Kim, Y. J., Kim, T. S. and Lee, S. K. 2005. Selection of virulent isolates of Fusarium circinatum and investigation of pitch canker severity of Pinus rigida and P. rigida $\times P$. taeda seed orchards in Jeju island. J. Kor. For. Sci. 94:402409. 